\title{
STUDY ON NONLOCAL MORPHOLOGICAL OPERATORS
}

\author{
Philippe Salembier \\ Signal Theory and Communications Department, \\ Technical University of Catalonia, Barcelona, Spain, philippe.salembier@upc.edu
}

\begin{abstract}
This paper studies nonlocal morphological operators. Following the strategy proposed for the Nonlocal means filter, these operators are based on a local and adaptive definition of the structuring element which is specified by searching for patches in the image that are similar to the one surrounding the pixel being filtered. After presenting the basic nonlocal morphological operators using generic structuring elements, we study their robustness in the presence of noise. Then, we illustrate the interest of some nonlocal morphological operators using flat structuring elements for denoising.
\end{abstract}

Index Terms - Nonlocal means, Nonlocal mathematical morphology, Adaptive structuring element.

\section{INTRODUCTION}

Adaptive mathematical morphology refers to morphological filtering techniques that adjust to the local context of the signal $[1,2,4-8]$. For $2 \mathrm{D}$ images, the adaptation is generally based on the intensity values of the signal as well as on the spatial position of the pixel to process. One way to obtain adaptive morphological operators is by defining a specific structuring element for each spatial position. Several approaches have been proposed and studied in the past $[1,2,6]$. In this paper, the strategy proposed in [3] to define the Nonlocal means filters is used to define a new class of adaptive morphological operators called Nonlocal morphological operators. The structuring elements of the resulting operators are specified for each pixel location by searching for patches in the image that are similar to the one surrounding the pixel being filtered. These operators provide interesting results for image filtering and noise cancelation.

This paper is organized as follows: the following section gives an overview of Nonlocal means filter. Then section 3 defines Nonlocal morphological operators with arbitrary gray level structuring element and studies their robustness in presence of noise. Section 4 discusses two possible definitions of nonlocal morphological operators with flat structuring elements and apply them for SAR images. Finally, section 5 is devoted to the conclusions.

\section{NONLOCAL MEANS FILTER}

Nonlocal means filters have been proposed in [3] mainly for denoising applications. The filtering idea consists in computing a weighted average of the input signal in a neighborhood $N_{n}$ :

$$
y[n]=N L M\{x[n]\}=\sum_{k \in N_{n}} w(n, k) x[k]
$$

where the weights $w(n, k)$ are defined by computing the similarity between a patch $P$ centered around the pixel $n$ to process and a patch around the pixel at position $k$. If the patch around the current pixel $n$ is very similar to the patch centered around $k$, then the weight $w(n, k)$ should be close to one. On the contrary, if both patches are very different, then the weight $w(n, k)$ should be close to zero. As can be seen, the weighted average takes into account mainly pixels that are surrounded by a patch that is similar to the one surrounding the pixel being processed. This is the key point explaining the robustness of this filter. The resulting filter is adaptive or translation-variant as $w(n, k)$ is in general not only function of $n-k$. (in this case, equ. (1) would define a convolution corresponding to a Linear Translation-Invariant system). In [3], the weights are defined as follows:

$$
w(n, k)=\frac{1}{Z} \exp \left(\frac{-1}{h^{2}} \sum_{m \in P} g(m)\|x[n-m]-x[k-m]\|^{2}\right)
$$

In this formula, the similarity between the patches centered around $n$ and $k$ is computed through a weighted Euclidean distance: $\sum_{m \in P} g(m)\|x[n-m]-x[k-m]\|^{2}$, where $m$ represents the indexes used to scan the patch $P$ and $g(m)$ is a Gaussian kernel giving higher (lower) weights to the central (outer) pixels of the patches. $Z$ is a normalizing constant ensuring that:

$$
\sum_{k \in N_{n}} w(n, k)=1
$$

Finally, $h$ is the filter parameter. If $h$ has a low (high) value, the filter will have a low (high) smoothing effect. 


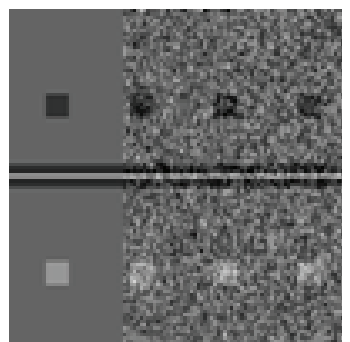

a)

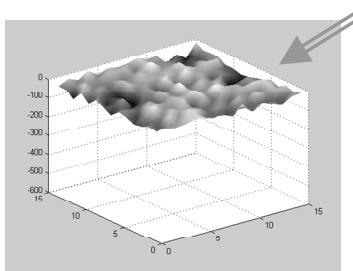

c)

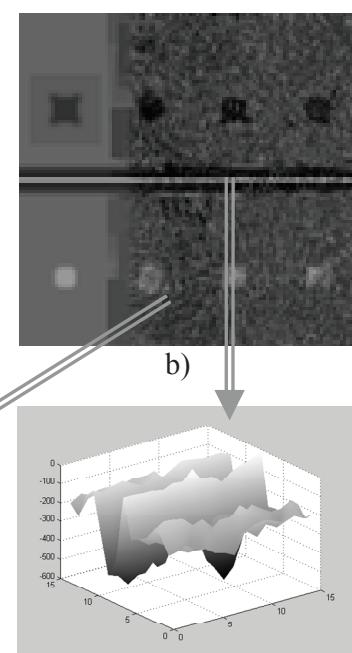

d)
Fig. 1: a) Original test image corrupted by Gaussian noise (center of the image) and Uniform noise (right part of the image), b) Nonlocal erosion, c) Shape of the structuring element in uniform areas, d) Shape of the structuring element in presence of structures

\section{NONLOCAL MORPHOLOGICAL OPERATORS WITH ARBITRARY STRUCTURING ELEMENTS}

Equ. (1) can be seen as a convolution where the value of the impulse response is computed and adapted to each pixel position. Consider now the equation defining a dilation with an arbitrary (that is, non flat) structuring element:

$$
y[n]=\delta\{x[n]\}=\bigvee_{k \in N_{n}}[w(n, k)+x[k]]
$$

where $\vee$ represents the supremum operation. If $w(n, k)$ is only function of $n-k$, then equ. (4) defines the classical Translation-Invariant dilation. Following the idea proposed in [3] for nonlocal means, we now assume that the weights $w(n, k)$ are derived from a similarity estimation between patches centered around $n$ and $k$.

In the case of the Nonlocal means filters, locations $k$ whose patches are very different from the one centered around $n$ should not influence the filter output. This is the reason why their associated weight is close to zero. In the case of the dilation, the weight is added, and not multiplied, to $x[k]$. Taking into account that the dilation is computed through a supremum operation, the locations $k$ whose patches are very different from the one centered around $n$, should be associated to a weight with a negative value of high magnitude, so that they have little influence in the output:

$$
w(n, k)=-\alpha \sum_{m \in P} g(m)\|x[n-m]-x[k-m]\|^{2}
$$

where $\alpha$ is the filter parameter. If $\alpha$ is close to zero, the structuring element is almost flat and corresponds to the area defined by $N_{n}$. If $\alpha$ has a high value, the dilation mainly computes the supremum of the pixels that have very similar patches around them. Equ. (4) and (5) define Nonlocal Morphological dilation. By duality, a Nonlocal morphological erosion can be defined by the following equation:

$$
y[n]=\varepsilon\{x[n]\}=\bigwedge_{k \in N_{n}}[w(n, k)+x[k]]
$$

where $\wedge$ represents the infimum. Based on these two basic operators, the following classical combinations can be defined: $\gamma()=.\delta(\varepsilon()$.$) used to remove maxima, \varphi()=.\varepsilon(\delta()$. used to remove minima, $\gamma(\varphi()$.$) and \varphi(\gamma()$.$) used to remove$ both minima and maxima and $(\gamma(\varphi())+.\varphi(\gamma())) /$.2 which is a self-dual operator removing both minima and maxima. Note that, even if we use the symbols $\gamma$ and $\varphi$ to highlight the conceptual similarity with morphological opening and closing, these operators are neither opening nor closing. Indeed, equ. (5) defines the weights from the input signal itself. As a result, the operators are not idempotent.

Fig. 1 illustrates the Nonlocal erosion. The original image (Fig. 1.a) is composed of a noise free area (left), an area corrupted by a Gaussian noise (center) and an area corrupted by uniform noise (right). Fig. 1.b shows the result of a nonlocal erosion with a neighborhood $N_{n}$ of $15 \times 15$ pixels and patches $P$ of $5 \times 5$ pixels. Fig. 1.c shows a typical structuring element of a non structured area of the image, whereas Fig. 1.d shows an example of structuring element belonging to a structured part of the image. As can be seen, the structuring element adapts well to the local image configuration. In particular, negative values of high magnitude are assigned to pixels that correspond to patches that are very dissimilar to the one being processed.

These operators have been used to remove the noise from the original image of Fig. 1.a. The results are shown in Fig. 2. More precisely, Fig. 2.a gives the results for the Nonlocal means filter and Fig. 2.b the results for the Nonlocal morphological operator $(\gamma \varphi+\varphi \gamma) / 2$. The rest of the figure shows intermediate results to compute the morphological operator. Analyzing the performances of the filters in the three areas of the image (left, center, right), it can be concluded that the Nonlocal morphological operator $(\gamma \varphi+$ $\varphi \gamma) / 2$ has very attractive properties in terms of noise cancelation, in particular for uniform noise (right section of the image) while preserving the contour of the image. 


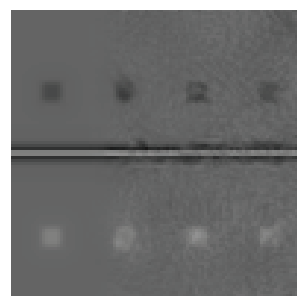

a)

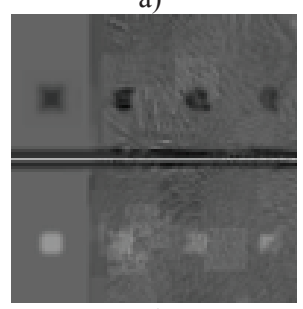

c)

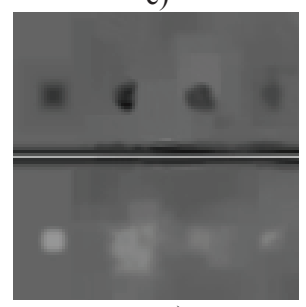

e)

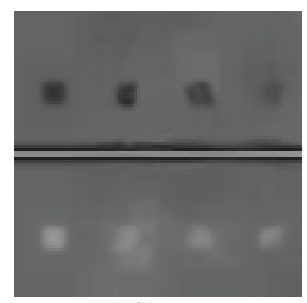

b)

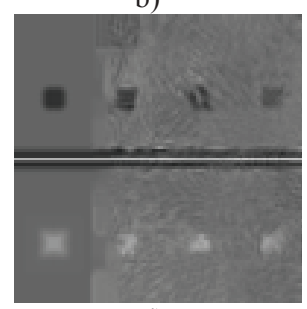

d)

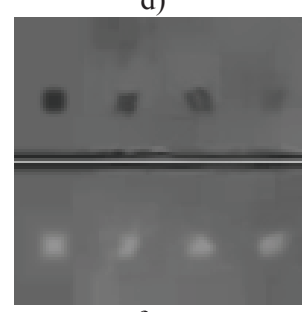

f)
Fig. 2: a) Nonlocal means, b) Nonlocal morphological operator: $(\varphi \gamma+\gamma \varphi) / 2$, c) $\gamma$, d) $\varphi$, e) $\varphi \gamma$, f) $\gamma \varphi$

In order to evaluate objectively the filters performances, Fig. 3 reports the PSNR as a function of the filters parameter ( $h$ for the Nonlocal means and $\alpha$ for the Nonlocal morphological operator $(\gamma \varphi+\varphi \gamma) / 2)$. As can be seen, the Nonlocal means filter exhibits very good performances for Gaussian noise. The Nonlocal morphological operator $(\gamma \varphi+$ $\varphi \gamma) / 2$ is slightly worse in the case of Gaussian noise but much better in the case of uniform noise.

\section{NONLOCAL MORPHOLOGICAL OPERATORS WITH FLAT STRUCTURING ELEMENTS}

In this section, we focus on Nonlocal morphological operators with flat structuring elements. A structuring element is flat if its samples can only take two values: zero or $-\infty$. In the nonlocal case, this means that the weights $w(n, k)$ can be either zero or $-\infty$. Equ. (4) becomes:

$$
y[n]=\delta\{x[n]\}=\bigvee_{k \in N_{n} \text { such that } w(n, k)=0} x[k]
$$

This means that the Nonlocal dilation consists in computing the supremum of input values that are surrounded by patches that are similar to the one surrounding the pixel being processed.

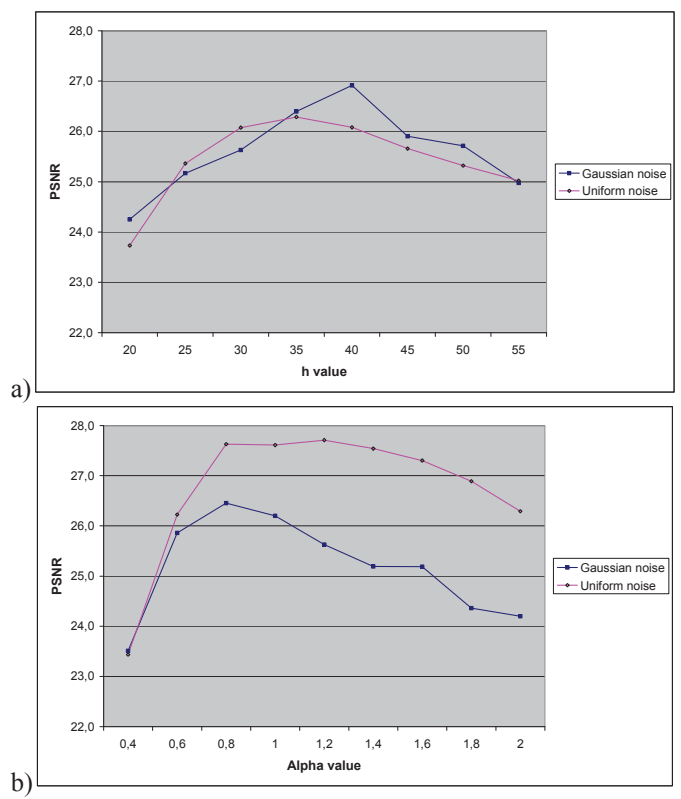

Fig. 3: a) Nonlocal Mean: PSNR as a function of the $h$ filter parameter, b) Nonlocal morphological operator $(\gamma \varphi+\varphi \gamma) / 2$ :

PSNR as a function of the $\alpha$ filter parameter

A simple strategy to define the flat structuring element is by thresholding the weights $w(n, k)$ as defined by Equ. (5).

Fig. 4 illustrates an example of filtering a Synthetic Aperture Radar (SAR) image. The original image is presented in Fig. 4.a and shows a highway surrounded by vegetation. Fig. 4.b shows the result applying the Nonlocal means filter and Fig. 4.c presents the result using the Nonlocal morphological operator $(\gamma \varphi+\varphi \gamma) / 2$ with flat structuring element. In this last case, the operator has a strong smoothing effect in unstructured areas of the image (bright areas in the lower left corner of the image) and a weak smoothing effect in structured parts of the image (the highway area). In fact, in unstructured areas, many patches are similar to the one surrounding the pixel being processed. Therefore, many weight values are above the threshold and the resulting structuring element involves many pixels. By contrast, in highly structured areas, dissimilarity of patches may be higher and the resulting structuring element involves a small number of pixels. Fig. 5 illustrates this idea showing, in gray level, the distribution of number of pixels involved in the structuring element. As can be seen, structuring elements involving a large number of pixels are mainly used in the lower left corner of the image. As a result, the smoothing strength of the filter depends on the type of image area that is being filtered.

To solve this issue and define a filter that has a constant number of pixels per structuring element, one can simply 


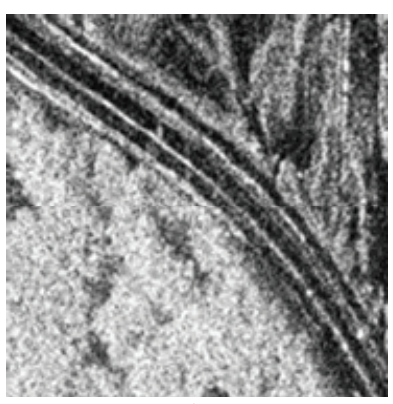

a)

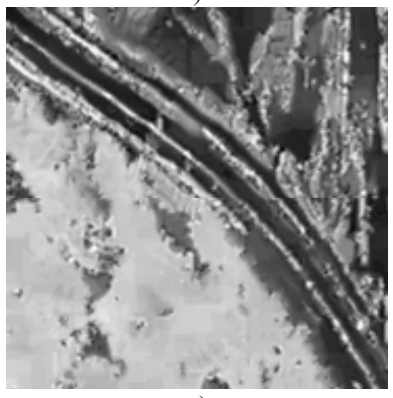

c)

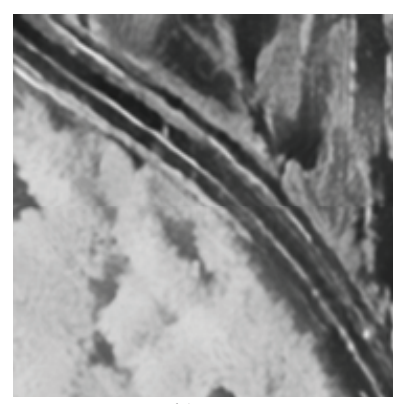

b)

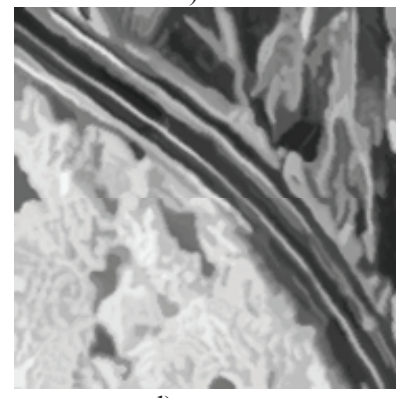

d)
Fig. 4: a) Original SAR image, b) Nonlocal mean, c) Nonlocal morphological operator $(\gamma \varphi+\varphi \gamma) / 2$ with flat structuring elements of variable size and d) Nonlocal morphological operator $(\gamma \varphi+$

$\varphi \gamma) / 2$ with flat structuring element of fixed size $(M=10)$. Note that in all cases, $N_{n}=15 \times 15$ pixels and $P=5 \times 5$ pixels.

include in the structuring element a fixed number $M$ of pixels corresponding to the $M$ most similar patches locations. This strategy is illustrated in Fig. 4.d. As can be seen, the approach provides a constant smoothing effect in the image. Moreover, as can be seen in Fig. 4, the contour preservation characteristics of this filter are better than the one provided by the Non local means filter.

\section{CONCLUSIONS}

This paper has investigated Nonlocal morphological operators. Following the strategy proposed for the Nonlocal means filter, these operators are based on a local and adaptive definition of the structuring element which is specified by searching for patches in the image that are similar to the one surrounding the pixel being filtered. After presenting the basic nonlocal morphological operators using generic structuring elements, their robustness in the presence of noise has been studied. It has been shown that these operators are quite robust in the presence of noise and also outperform the Nonlocal means filters for certain type of noise distribution. Then, two strategies to define nonlocal morphological operators with flat structuring elements have been proposed. The one selecting a constant number of pixels per structuring element seems to offer good tradeoff between smoothing and preserving contours. This feature has been illustrated on SAR images.

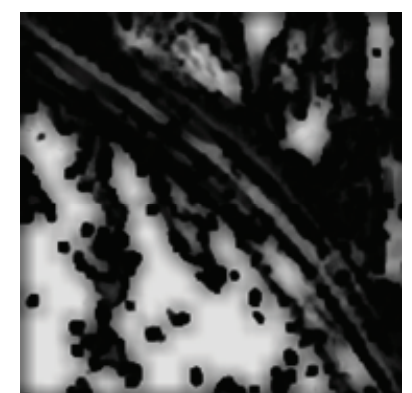

Fig. 5: Example of distribution of the structuring element size in Fig. 4.c.

\section{ACKNOWLEDGMENTS}

This work was partially supported by the Spanish Ministerio de Educación y Ciencia, under project TEC200766858/TCM: Provec.

\section{REFERENCES}

[1] N. Bouaynaya, M. Charif-Chefchaouni, D. Schonfeld, Theoretical foundations of spatially-variant mathematical morphology - Part I: Binary images, IEEE Transactions on Pattern Analysis and Machine Intelligence, vol. 30, pp. 823836, 2008.

[2] N. Bouaynaya, D. Schonfeld, Theoretical foundations of spatially-variant mathematical morphology - Part II: Graylevel images, IEEE Transactions on Pattern Analysis and Machine Intelligence, vol. 30, pp. 837-850, 2008.

[3] A. Buades, B. Coll, J.M. Morel, Image Denoising By NonLocal Averaging, Proceedings of the IEEE International Conference on Acoustics, Speech, and Signal Processing 2005, ICASSP '05, Volume 2, March 18-23, 2005 Page(s):25 - 28.

[4] J. Debayle, J. C. Pinoli. General Adaptive Neighborhood Image Processing - Part I: Introduction and Theoretical Aspects. Journal of Mathematical Imaging and Vision, 25(2):245--266, Sept. 2006.

[5] J. Debayle, J. C. Pinoli. General Adaptive Neighborhood Image Processing - Part II: Practical Application Examples. Journal of Mathematical Imaging and Vision, 25(2):267--284, Sept. 2006.

[6] P. Salembier. Structuring element adaptation for morphological filters. Journal of Visual Communication and Image Representation, 3(2):115-136, June 1992.

[7] F.Y. Shih, S. Cheng, Adaptive Mathematical Morphology for Edge Linking, International Journal of Information Sciences Informatics and Computer Science, vol. 167, pp. 9-21, Dec. 2004.

[8] C. Vachier, F. Meyer. News from viscous Land. International Symposium on Mathematical Morphology, pages 189--200, Oct. 2007, Rio de Janeiro, Brazil. 